\title{
Stellar velocity dispersion and dynamical mass of the ultra diffuse galaxy NGC 5846_UDG1 from the keck cosmic web imager
}

\author{
Duncan A. Forbes, ${ }^{1 \star}$ Jonah S. Gannon, ${ }^{1}$ Aaron J. Romanowsky ${ }^{\circledR},{ }^{2,3}$ Adebusola Alabi ${ }^{\circledR}, 3$ \\ Jean P. Brodie, ${ }^{1,3}$ Warrick J. Couch ${ }^{1}$ and Anna Ferré-Mateu ${ }^{\circledR 1,4}$ \\ ${ }^{1}$ Centre for Astrophysics \& Supercomputing, Swinburne University, Hawthorn, VIC 3122, Australia \\ ${ }^{2}$ Department of Physics \& Astronomy, San José State University, San Jose, CA 95192, USA \\ ${ }^{3}$ University of California Observatories, 1156 High St, Santa Cruz, CA 95064, USA \\ ${ }^{4}$ Institut de Ciencies del Cosmos (ICCUB), Universitat de Barcelona (IEEC-UB), E-02028 Barcelona, Spain
}

Accepted 2020 October 20. Received 2020 October 6; in original form 2020 August 12

\begin{abstract}
The ultra diffuse galaxy in the NGC 5846 group (NGC 5846_UDG1) was shown to have a large number of globular cluster (GC) candidates from deep imaging as part of the VEGAS survey. Recently, Müller et al. published a velocity dispersion, based on a dozen of its GCs. Within their quoted uncertainties, the resulting dynamical mass allowed for either a dark matter free or a dark-matter-dominated galaxy. Here, we present spectra from KCWI that reconfirms membership of the NGC 5846 group and reveals a stellar velocity dispersion for UDG1 of $\sigma_{\mathrm{GC}}=17 \pm 2 \mathrm{~km} \mathrm{~s}^{-1}$. Our dynamical mass, with a reduced uncertainty, indicates a very high contribution of dark matter within the effective radius. We also derive an enclosed mass from the locations and motions of the GCs using the tracer mass estimator, finding a similar mass inferred from our stellar velocity dispersion. We find no evidence that the galaxy is rotating and is thus likely pressure supported. The number of confirmed GCs, and the total number inferred for the system $(\sim 45)$, suggests a total halo mass of $\sim 2 \times 10^{11} \mathrm{M}_{\odot}$. A cored mass profile is favoured when compared to our dynamical mass. Given its stellar mass of $1.1 \times 10^{8} \mathrm{M}_{\odot}$, NGC 5846_UDG1 appears to be an ultra diffuse galaxy with a dwarf-like stellar mass and an overly massive halo.
\end{abstract}

Key words: galaxies: haloes-galaxies: kinematics and dynamics-galaxies: star clusters: general.

\section{INTRODUCTION}

Ultra diffuse galaxies (UDGs) were first identified using a novel telescope (actually a collection of multiple cameras called The Dragonfly Array) optimized for detecting low surface brightness objects by van Dokkum et al. (2015). Further deep imaging has revealed UDGs in all environments, although they are more common in clusters (e.g. Janssens et al. 2019). They are defined to have halflight radii $\left(\mathrm{R}_{\mathrm{e}}\right)>1.5 \mathrm{kpc}$ and central surface brightnesses $\left(\mu_{0}\right)>24$ mag $\operatorname{arcsec}^{-2}$ in the $g$ band. With stellar masses $\left(\mathrm{M}_{*}\right)$ of around $10^{8}$ $\mathrm{M}_{\odot}$, they have half-light radii comparable to the Milky Way but the stellar content of dwarfs. However, perhaps their most remarkable property is that some appear to have overmassive (Beasley et al. 2016; van Dokkum et al. 2016; Forbes et al. 2019) and even undermassive dark matter haloes (Danieli et al. 2019; van Dokkum et al. 2019a). Total halo masses are either inferred from counting their globular clusters (GCs) and applying the scaling relation for normal galaxies (Burkert \& Forbes 2020) or they are inferred from measured dynamical masses and scaled assuming a mass profile (Wasserman et al. 2019; Gannon et al. 2020).

Although the best-studied UDG with an overmassive halo, Dragonfly 44 (DF44) in the Coma cluster, obeys the GC number versus halo mass relation, the undermassive halo UDG DF2 in the

^E-mail: dforbes@swin.edu.au
NGC 1052 group does not (although in this case the compact sources seen may not all be GCs). The alternative approach is to measure the velocity dispersion of a UDG and calculate its dynamical mass. Due to observational challenges, this has only been carried out for a small number of UDGs by either measuring the integrated properties of the galaxy stars or from the collective motions of their GCs. The number of UDGs with both stellar and GC orbit-based velocity dispersions in the literature is currently only two (i.e. NGC 1052DF2 and VCC 1287). Clearly, more UDGs need to have their stellar velocity dispersions measured in order to test whether the GC-derived values, often based on very small samples, are reliable.

Integral field units (IFUs) optimized for spatially resolved spectroscopy of low surface brightness objects are now available on 8-10 m class telescopes. They include KCWI on Keck, MUSE on VLT, and Megara on GTC. Each has its own comparative advantage for studying UDGs: KCWI has an excellent blue response and can operate at high spectral resolution; MUSE has a large field of view $\left(\sim 1 \times 1 \mathrm{arcmin}^{2}\right)$ that allows for on-chip sky subtraction of more distant UDGs, Megara offers high spectral resolution and dedicated fibres for sky subtraction. Even with long exposure times on such large telescopes, these IFUs are often used in 'light-bucket' mode where all spaxels are collapsed to give a single kinematic measure for the galaxy.

NGC 5846_UDG1 was first identified by Mahdavi, Trentham \& Tully (2005) as a 'very low surface brightness galaxy' that y dubbed NGC 5846-156. From deeper VEGAS imaging (Spavone et al. 2017), 
Forbes et al. (2019) determined it had the properties of a UDG if at the distance of the NGC 5846 group. The imaging also revealed a system of at least 20 compact objects (likely to be GCs). More recently, Müller et al. (2020; hereafter M20), using MUSE on the VLT, measured a recession velocity placing it in the NGC 5846 group. They also confirmed the association of a dozen GCs with the UDG (which they refer to as MATLAS J15052031+0148447). From the motions of these objects, they measured a velocity dispersion and determined an enclosed dynamical mass within the de-projected halflight radius (following Wolf et al. 2010) of $1.8_{-1.5}^{+3.7} \times 10^{8} \mathrm{M}_{\odot}$. Using their luminosity, this gave a mass-to-light ratio in the $V$ band of $4.2_{-3.4}^{+8.6}$. Their lower limit is comparable to the galaxy's stellar M/L and draws comparison to the two UDGs in the NGC 1052 group that appear to lack dark matter (van Dokkum et al. 2019a), whereas their higher limit suggests a substantial dark matter halo as seen in some other UDGs (e.g. Gannon et al. 2020). As their GC sample is subject to small number statistics (see Laporte, Agnello \& Navarro 2019) a high precision stellar velocity dispersion is desirable in order to better define the mass-to-light ratio of NGC 5846_UDG1 and hence test whether the populous GC system seen in the imaging of Forbes et al. (2019) is associated with a massive halo as might be expected.

Here, we present spectra obtained for NGC 5846_UDG1 using KCWI on the Keck telescope from which we measure its stellar velocity dispersion. KCWI has the advantage of superior spectral resolution over MUSE, i.e. $\sigma_{\text {inst }}=13 \mathrm{~km} \mathrm{~s}^{-1}$ for KCWI versus $45 \mathrm{~km} \mathrm{~s}^{-1}$ for MUSE. We also discuss other UDGs for which both stellar and GC-orbit based velocity dispersions are available. We search for signs of rotation in the main body of the galaxy and measure the recession velocity for two bright GCs. We derive a dynamical mass and compare it to an inferred total halo mass for NGC 5846_UDG1.

\section{OBSERVATIONS AND DATA REDUCTION}

NGC 5846_UDG1 was observed with KCWI (Martin et al. 2010) on the Keck II telescope under program N061 on the nights of 2019 March 30, May 1, and May 29. We employed the BH3 grating centred at $5110 \AA$ with a full wavelength range of $4850-5350 \AA$. The spectral resolution is $\sigma_{\text {inst }}=13 \mathrm{~km} \mathrm{~s}^{-1}$. We used the medium slicer with a field of view of $16 \times 20 \operatorname{arcsec}^{2}$, with the long axis of KCWI orientated roughly in the NE-SW direction with a PA of $60^{\circ}$ (see Fig. 1). Our field of view extends to around 50 percent of the half-light radius of $17.7 \pm 0.5 \mathrm{arcsec}$ (Forbes et al. 2019). Conditions were dark but some thin cloud was present. The total time on the science target was $21900 \mathrm{~s}$. We alternated on-target observations with offset positions, obtaining a total of $19200 \mathrm{~s}$ on sky. A standard star was observed with the same set-up and used for flux calibration. The data were reduced following the method of Gannon et al. (2020), i.e. we ran the standard KCWI data but skipped stage 5 (standard sky subtraction). Following this, we perform the data correction described in appendix A of Gannon et al. (2020) and sky subtraction using our PCA-based routine. After application of the corresponding barycentric corrections, we median combine the sky-subtracted spectra.

After masking the two brightest compact sources present (the remaining compact sources in the field of view contribute $\leq 6$ per cent of the total flux), we extract a spectrum for the galaxy. In Fig. 2, we show the final spectrum obtained by collapsing all spectra across the galaxy. It has an $\mathrm{S} / \mathrm{N}$ of $\sim 20$ per $\AA$ in the continuum. We also show the best fit to the spectrum using pPXF (Cappellari 2017) and the resulting residuals. In order to fully explore the possible choices of PPXF input parameters, we fit a wide ranging selection

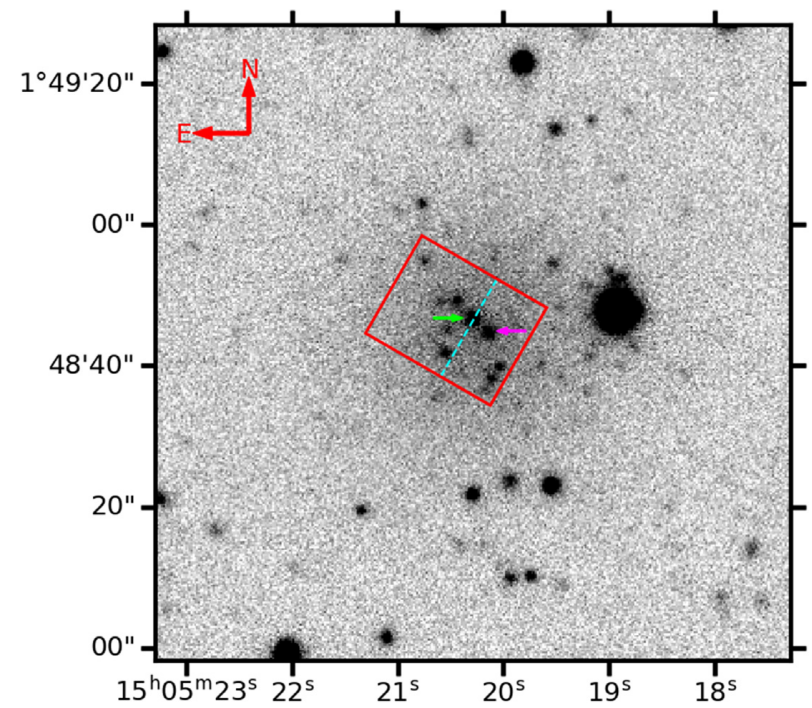

Figure 1. Image of NGC 5846_UDG1 with overlay showing KCWI footprint. The solid red overlay shows the orientation of KCWI's $16 \times 20 \operatorname{arcsec}^{2}$ field of view at PA $=60^{\circ}$. The dashed cyan line shows the axis used to separate the NE from SW regions of the galaxy. The green arrow points to globular cluster GC9 and the purple arrow arrow to GC10. We extract spectra of the galaxy and the two GCs separately. The galaxy has a half-light radius of $17.7 \pm 0.5$ arcsec with its centre near GC9 (which may be the stellar nucleus).

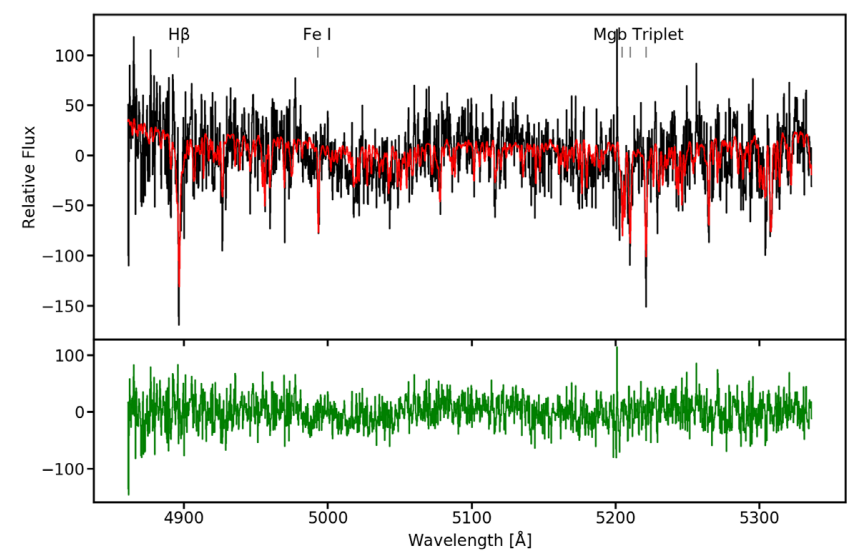

Figure 2. Our final spectrum for NGC 5846_UDG1 with a representative stellar population model fit. Our final spectrum (black) is overplotted with a representative fit (red) from our exhaustive pPXF fitting with the Coelho (2014) synthetic stellar library. Residuals of the fit (green) are shown in the lower panel. $\mathrm{H} \beta, \mathrm{Fe} \mathrm{I}$, and the $\mathrm{Mg}$ b triplet have been labelled. Based on our fitting of the NGC 5846_UDG1 spectrum we recover a velocity dispersion of $\sigma_{*}=17 \pm 2 \mathrm{~km} \mathrm{~s}^{-1}$.

of input parameters as described in Gannon et al. (2020). We do so using both the Coelho (2014) synthetic stellar library and an observation of the Milky Way GC M3. We observed M3 with the same KCWI instrumental configuration, although with a slightly lower central wavelength to allow for the predicted disparity in recessional velocities between M3 and the target. We also fit five different spectral regions of the final spectrum to ensure our final value is not driven by any particular spectral region. Fitting with both the Coelho (2014) and M3 templates displays good convergence in both recessional velocity and velocity dispersion. Our final values for recessional velocity and velocity dispersion are $2167 \pm 2 \mathrm{~km} \mathrm{~s}^{-1}$ and $\sigma_{*}=17 \pm 2 \mathrm{~km} \mathrm{~s}^{-1}$, respectively. 
Table 1. NGC 5846_UDG1 properties.

\begin{tabular}{lc}
\hline Property & Value \\
\hline RA (J2000) & $15: 05: 20$ \\
Dec. $(\mathrm{J} 2000)$ & $01: 48: 47$ \\
Velocity $\left(\mathrm{km} \mathrm{s}^{-1}\right)$ & $2167 \pm 2$ \\
Distance $(\mathrm{Mpc})$ & 24.89 \\
$\mathrm{R}_{\mathrm{e}}(\mathrm{kpc})$ & $2.14 \pm 0.06$ \\
$\mathrm{Sersic}$ & 0.68 \\
$\mathrm{M}_{V}(\mathrm{mag})$ & $-14.5 \pm 0.2$ \\
$g-i(\mathrm{mag})$ & $\sim 1$ \\
$\mu_{0}\left(\mathrm{mag} \mathrm{arcsec}^{-2}\right)$ & $24.8 \pm 0.1$ \\
$\mathrm{M}_{*}\left(\mathrm{M}_{\odot}\right)$ & $1.1 \times 10^{8}$ \\
$\sigma_{*}\left(\mathrm{~km} \mathrm{~s}^{-1}\right)$ & $17 \pm 2$ \\
$\mathrm{M}_{1 / 2}\left(10^{8} \mathrm{M}_{\odot}\right)$ & $5.46 \pm 1.3$ \\
$\mathrm{M}_{1 / 2} / \mathrm{L}_{1 / 2}$ & $19.9 \pm 5.7$ \\
$\mathrm{GCs}$ & $\sim 45$ \\
\hline Note. Values from this work or Forbes et & $(2019)$
\end{tabular}

\section{RESULTS}

The measured properties of NGC 5846_UDG1 from this work and the deep imaging study of Forbes et al. (2019) are summarized in Table 1 .

Our measurement of the recession velocity $\left(2167 \pm 2 \mathrm{~km} \mathrm{~s}^{-1}\right)$ is similar to that quoted by M20 in their table 1, i.e. $2156 \pm 9.4 \mathrm{~km} \mathrm{~s}^{-1}$. This velocity places NGC 5846_UDG1 in the NGC 5846 group, which has a distance of $25 \mathrm{Mpc}$ based on a mean of the surface brightness fluctuation and Fundamental Plane distances (Tully et al. 2013). Here, we assume the same distance as used by Forbes et al. (2019) and Spavone et al. (2017) of 24.89 Mpc (M20 assumed a distance of $26.3 \mathrm{Mpc}$.)

We also extract spectra for the two bright compact sources in the KCWI field of view. Using the naming convention from Forbes et al. (2019), they are GC10 and GC9. We measure a recession velocity for GC10 of $2163 \pm 3 \mathrm{~km} \mathrm{~s}^{-1}$ compared to $2147 \pm 8 \mathrm{~km} \mathrm{~s}^{-1}$ by M20 (which they call GC5), and for GC9 we measure $2166 \pm 3 \mathrm{~km} \mathrm{~s}^{-1}$ compared to $2147 \pm 5 \mathrm{~km} \mathrm{~s}^{-1}$ in M20 (which they call GC6). We note that GC9 (the brightest and centrally located source) has a similar velocity to the host galaxy $\left(2167 \pm 2 \mathrm{~km} \mathrm{~s}^{-1}\right)$ and may be its stellar nucleus (see also Forbes et al. 2019).

\subsection{Kinematics}

We measure a stellar velocity dispersion within our KCWI field of view of $\sigma_{*}=17 \pm 2 \mathrm{~km} \mathrm{~s}^{-1}$. Our uncertainty is the error on the mean value from fits using different stellar libraries, templates, and spectral regions as described in Section 2. Within the joint uncertainties, the GC-based velocity dispersion of $\mathrm{M} 20, \sigma_{\mathrm{GC}}=$ $9.4_{-5.4}^{+7.0} \mathrm{~km} \mathrm{~s}^{-1}$ is consistent with our value. M20 measured old ages and low metallicities typical of MW GCs, although we question their quoted levels of uncertainty for some individual GCs. For example, they quoted an age of $9.1 \mathrm{Gyr}$ with an accuracy of +1.6 and $-2.6 \mathrm{Gyr}$ for a a GC spectrum with an $\mathrm{S} / \mathrm{N}$ per pixel of only 3.7 . We note that the work of Conroy et al. (2018) suggests that an $\mathrm{S} / \mathrm{N}>40 \AA$ is needed to measure age and metallicity to within 0.1 dex Unfortunately, they did not provide spectra nor their model fit in their paper. We also note that if they had used the galaxy velocity as a prior in their MCMC analysis of the GC system, then they may have derived a slightly higher velocity dispersion (see their fig. 6).

In Fig. 3, we compare velocity dispersion measures from stars with those from GCs for NGC 5846_UDG1 and for other galaxies in the literature. There are only two other UDGs for which both stellar

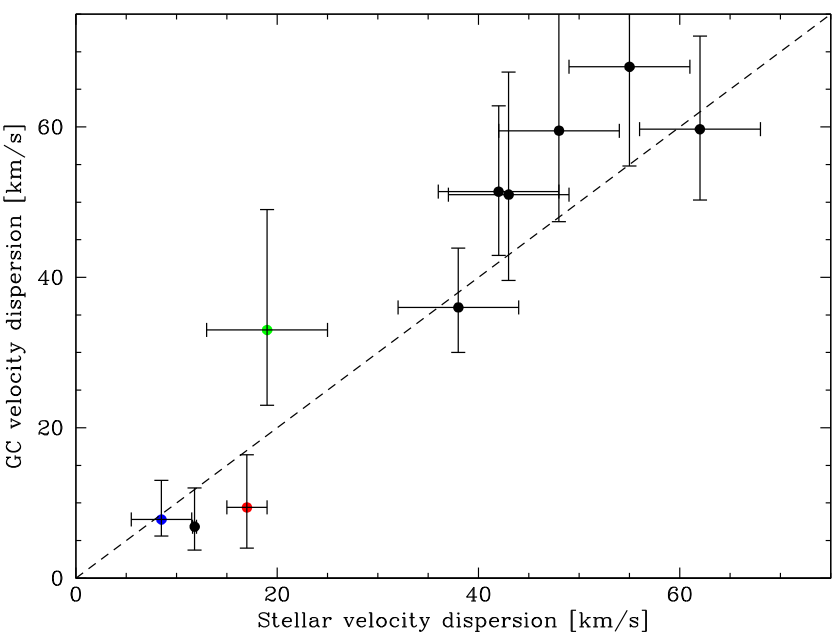

Figure 3. Comparison of velocity dispersions from stars and globular cluster systems. The dashed line is not a fit but a unity line. Ultra diffuse galaxies are shown by coloured symbols (red for NGC 5846_UDG1, green for VCC 1287, and blue for DF2). Other early-type galaxies (from Laporte et al. 2019 and Fahrion et al. 2020) are shown in black.

Table 2. UDG velocity dispersions.

\begin{tabular}{lccc}
\hline Name & $\begin{array}{c}\sigma_{*} \\
\left(\mathrm{~km} \mathrm{~s}^{-1}\right)\end{array}$ & $\begin{array}{c}\sigma_{\mathrm{GC}} \\
\left(\mathrm{km} \mathrm{s}^{-1}\right)\end{array}$ & Ref. \\
\hline NGC 5846_UDG1 & $17 \pm 2$ & $9.4_{-5.4}^{+7.0}$ & 1,2 \\
VCC 1287 & $19 \pm 6$ & $33_{-10}^{+16}$ & 3,4 \\
NGC 1052-DF2 & $8.5_{-3.1}^{+2.3}$ & $7.8_{-2.2}^{+5.2}$ & 5,6 \\
\hline
\end{tabular}

Note. 1 This work, 2 Müller et al. (2020), 3 Gannon et al. (2020), 4 Beasley et al. (2016), 5 Danieli et al. (2019), 6 van Dokkum et al. (2018).

and GC-based velocity dispersions exist, i.e. VCC 1287 (Gannon et al. 2020) and NGC 1052-DF2 (Danieli et al. 2019). For these galaxies, and NGC 5846_UDG1, we summarize their values from the two methods in Table 2. For DF2, both low velocity dispersions are very consistent with each other. For NGC 5846_UDG1, the stellar velocity dispersion is higher than the GC one, whereas for VCC 1287 it is lower than the GC one, although in both cases the values are consistent within the quoted joint uncertainties. The stellar velocity dispersions listed in Table 2 have lower quoted uncertainties than those from GCs. We also include the Fornax dSph galaxy in Fig. 3 with the GC velocity dispersion of four GCs from Laporte et al. (2019) and the stellar velocity dispersion from McConnachie et al. (2012). In addition, we include some dwarf early-type galaxies from the Fornax 3D survey (Fahrion et al. 2020). We do not expect a perfect match between the stellar and GCbased velocity dispersions given differences in spatial coverage, anisotropies, tracer distributions etc. However, within the quoted uncertainties, velocity dispersions measured from GC systems and integrated stars for these low-mass galaxies are generally consistent with each other. One notable exception is VCC 1287 that has a much reduced GC velocity dispersion of $\sigma_{\mathrm{GC}}=21 \mathrm{~km} \mathrm{~s}^{-1}$ if the velocity outlier were removed (see Gannon et al. 2020). We note that for large early-type galaxies with rich GC systems, the two velocity dispersion measures are well correlated on a one-to-one basis (Pota et al. 2013). 


\subsection{Masses}

The absolute magnitude of NGC 5846_UDG1 from Forbes et al. (2019) is $\mathbf{M}_{V}=-14.5$. Adjusting to our adopted distance of 24.89 Mpc, M20 measured a magnitude $\mathrm{M}_{V}=-14.88$, whereas Mahdavi et al. (2005) measured -14.0 (assuming a typical UDG colour $V-R$ $=0.3$; Forbes et al. 2020). Thus, our magnitude lies between these two published values. Our total luminosity, $\mathrm{L}_{V}=0.55 \times 10^{8} \mathrm{M}_{\odot}$, translates to a total stellar mass of $\mathrm{M}_{*}=1.1 \times 10^{8} \mathrm{M}_{\odot}$ for a stellar $\mathrm{M} / \mathrm{L}_{V}$ ratio $=2$ (M20 have confirmed that NGC 5846_UDG1 is old and metal-poor).

We follow Wolf et al. (2010) to calculate the enclosed dynamical mass. The Wolf et al. formula minimizes the effect of non-isotropic orbits on the derived mass, while assuming a pressure-supported system (see Section 3.3 below). We assume that our velocity dispersion measurement is representative of the mean value within the de-projected half-light radius of $r_{1 / 2}=4 / 3 \times R_{e}=2.85 \mathrm{kpc}$. If the velocity dispersion profile is actually rising with radius, as seen in DF44 (van Dokkum et al. 2019b), then the true enclosed mass will be greater. The Wolf, or half, mass is $\mathrm{M}_{1 / 2}=930 \sigma^{2} R_{\mathrm{e}} \sqrt{b / a}$, where the ellipticity $b / a=0.9$ from M20. We derive the mass within the deprojected half-light radius to be $\mathrm{M}_{1 / 2}=5.46( \pm 1.3) \times 10^{8}$ $\mathrm{M}_{\odot}$. From the luminosity above, the resulting mass-to-light ratio is $\mathrm{M}_{1 / 2} / \mathrm{L}_{1 / 2}=19.9 \pm 5.7$.

M20 applied the Wolf et al. formula to their GC-based velocity dispersion (assuming the GC system had the same effective size as the galaxy half-light radius). They quoted a half mass of $\mathrm{M}_{1 / 2}=$ $1.8_{-1.5}^{+3.7} \times 10^{8} \mathrm{M}_{\odot}$. The main reason for their lower half mass is the lower velocity dispersion of their GCs compared to the integrated stars of the gaalxy. They also derived a mass-to-light ratio $\mathrm{M}_{1 / 2} / \mathrm{L}_{1 / 2}$ $=4.2_{-3.4}^{+8.6}$. Using their mass and our luminosity above, this ratio would rise to 6.5 .

In Fig. 4 , we show the half mass $\left(M_{1 / 2}\right)$ to half-light $\left(L_{1 / 2}\right)$ ratios for UDGs and other objects from the literature. For three UDGs, we show $\mathrm{M}_{1 / 2}$ estimates based on both stellar velocity dispersions and GC-based ones. For other galaxies, including two well-studied UDGs (DGSAT I; Martín-Navarro et al. (2019) and DF44; van Dokkum et al. 2019b), only mass-to-light ratios from stellar velocity dispersions are available. The plot shows the well-known U-shape for normal galaxies (from Wolf et al. 2010 and Forbes et al. 2011) with UDGs mostly scattering above the trend to higher mass-tolight ratios. The exceptions are NGC 1052-DF2 (for which both GC and stellar derived masses indicate little or no dark matter) and the GC based mass for NGC 5846_UDG1 from M20. For a discussion of aperture effects on the mass-to-light we refer the reader to van Dokkum et al. (2019a).

An alternative approach for calculating the enclosed mass is to use the tracer mass estimator (TME) of Watkins, Evans \& An (2010) based on the positions and velocities of the GCs. The TME has been used successfully by Alabi et al. (2016) to measure the enclosed mass within $5 \mathrm{R}_{\mathrm{e}}$ for the early-type galaxies in the SLUGGS survey using samples of tens to hundreds of GCs. Based on the virial theorem, the TME requires assumptions regarding the tracer distribution, gravitational potential, and orbits quantified by $\gamma, \alpha$, and $\beta$, respectively. van Dokkum et al. (2017) examined the radial distribution of GCs in two GC-rich UDGs (DF44 and DFX1). They found that the distribution was more extended than the galaxy light and could be fitted by a Sersic profile with $n \sim 3$. The profile (their fig. 2) can be approximated by a power-law slope of $\sim 1$ within $\mathrm{R}_{\mathrm{e}}$ and hence a deprojected 3D slope of around 2. Thus, we adopt $\gamma=$ 2 . For the potential, we explore the range $-1<\alpha<1$ noting that an isothermal potential $(\alpha=0)$ may be appropriate for low surface brightness galaxies (de Blok \& Bosma 2002). We allow orbits to range from mildly radial $(\beta=+0.5)$ to tangential $(\beta=-0.5)$, with isotropic orbits having $\beta=0$. Applying the TME to the GCs in M20, we derive a dynamical mass enclosed within the outermost GC (i.e. $2.2 \mathrm{kpc})$ of $3.48( \pm 0.84) \times 10^{8} \mathrm{M}_{\odot}$, with the uncertainty given by the range in potential and orbital anisotropy described above. Our TME dynamical mass is larger than the GC-based half mass, but agrees within the uncertainties. Allowing for a reasonable range in $\gamma$ would increase the uncertainty to around \pm 50 per cent; where assuming a lower (higher) value of $\gamma$ would lead to a reduced (increased) TME mass. The resulting mass-to-light ratio from our TME analysis is $\mathrm{M}_{1 / 2} / \mathrm{L}_{1 / 2}=12.7 \pm 3.7$.

Forbes et al. (2019) detected $20 \mathrm{GC}$ candidates associated with NGC 5846_UDG1. If we assume the same mean GC luminosity function turnover magnitude (i.e. $\mathrm{M}_{V}=-7.3$ ) and width as found by Miller \& Lotz (2007) for dE galaxies in the Virgo cluster, then the inferred total GC population is around 45. For this count, the Burkert $\&$ Forbes (2020) relation between the number of GCs and the halo mass implies a total halo mass of $\sim 2 \times 10^{11} \mathrm{M}_{\odot}$. Given that there are several unknowns in translating the detection of GC candidates into an estimate of the total GC system, it is useful to note that M20 confirmed at least a dozen GCs associated with NGC 5846_UDG1. This gives a lower limit on the total halo mass with this relation, of $0.6 \times 10^{11} \mathrm{M}_{\odot}$. Both of these total halo mass estimates far exceed the total stellar mass of $1.1 \times 10^{8} \mathrm{M}_{\odot}$. For comparison, M33 has the same inferred total halo mass of $2 \times 10^{11} \mathrm{M}_{\odot}$ (Seigar 2011) but its stellar mass is some $50 \times$ larger. Thus, NGC 5846_UDG1 can be described as having a dwarf-like stellar mass with an overly massive dark matter halo.

Assuming a total halo mass of $2 \times 10^{11} \mathrm{M}_{\odot}$ as described above, we can compare our half mass and TME mass with different halo mass profiles. We chose three profiles, namely the universal NFW profile (Navarro, Frenk \& White 1996), the mass profile resulting from the dwarf galaxy simulations of Di Cintio et al. (2014), which resembles a core-NFW profile, and the observationally motivated mass profile for dwarf galaxies from Burkert (1995). For details of the NFW and Di Cintio et al. profiles, see the appendix of Di Cintio et al. (2014). We show these three profiles, with the same total halo mass, compared to our half and TME masses measured at small radii in Fig. 5. The plot shows that our mass measurements lie between the Di Cintio and Burkert mass profiles for a total halo mass as given by the total number of GCs. This suggests that NGC 5846_UDG1 may be better represented by a core mass profile as predicted in some simulations (e.g. Carleton et al. 2019) than a cuspy one. However, we cannot, at this stage, rule out other mass profiles and/or different total halo masses. Similar conclusions, favouring a cored mass profile, were reached by Gannon et al. (2020) for VCC 1287, and by Wasserman et al. (2019) for DF44.

\subsection{Does NGC 5846_UDG1 Rotate?}

An assumption of both the half mass and the TME mass calculations is that the system is dominated by random motions, and not bulk rotation. The GC system of NGC 5846_UDG1 shows some evidence for rotation (see fig. 9 in M20). However, after fitting a sine function and solving for a variable rotation axis and rotation amplitude, via an MCMC analysis, M20 could not confirm (nor rule out) bulk rotation of the GC system. A rotational contribution to the velocity would scale as $\left(V_{\text {rot }} / \sin i\right)^{2}$ and so any correction to the half mass or TME mass would be highly dependent on the unknown inclination $i$. We note that the galaxy itself appears rather circular on the sky. 


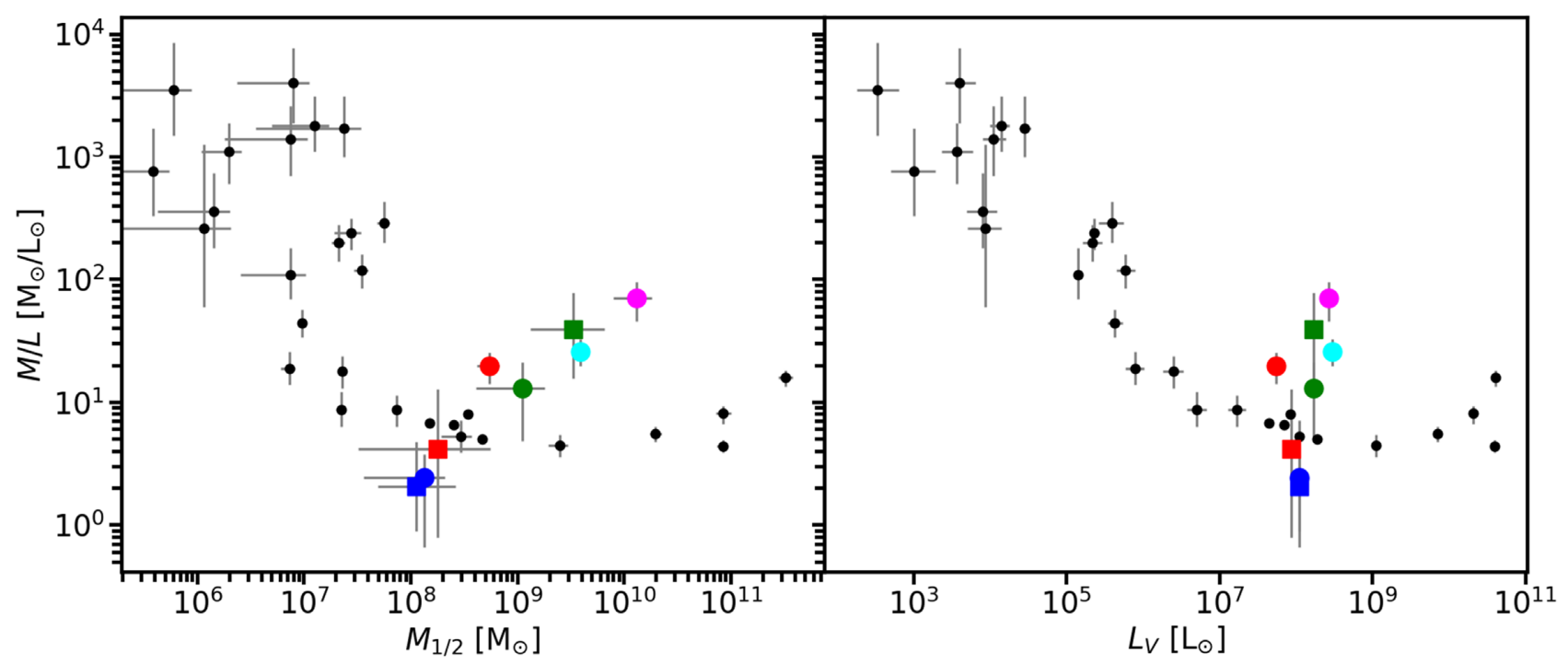

Figure 4. Dynamical mass-to-light ratio $\left(\mathrm{M}_{1 / 2} / \mathrm{L}_{1 / 2}\right)$ versus dynamical mass within the deprojected half-light radius (left) and total $\mathrm{V}$ band luminosity (right). Ultra diffuse galaxies are shown by coloured symbols (red for NGC 5846_UDG1, green for VCC 1287, and blue for DF2, cyan for DF44, and magenta for DGSAT I). For NGC 5846_UDG1, VCC 1287, and DF2 the plots show the mass-to-light ratio derived from GC (the squares) and stellar velocity dispersions (the circles). For NGC 5846_UDG1, we show mass and luminosity taken directly from M20 (the red square) and this work (the red circle). The black symbols show Local Group dwarfs and other pressure-supported galaxies from Wolf et al. (2010) and Forbes et al. (2011). Both distributions have a local minimum around $10^{9}$ in solar units, with most UDGs found above the distribution of normal galaxies with higher mass-to-light ratios.

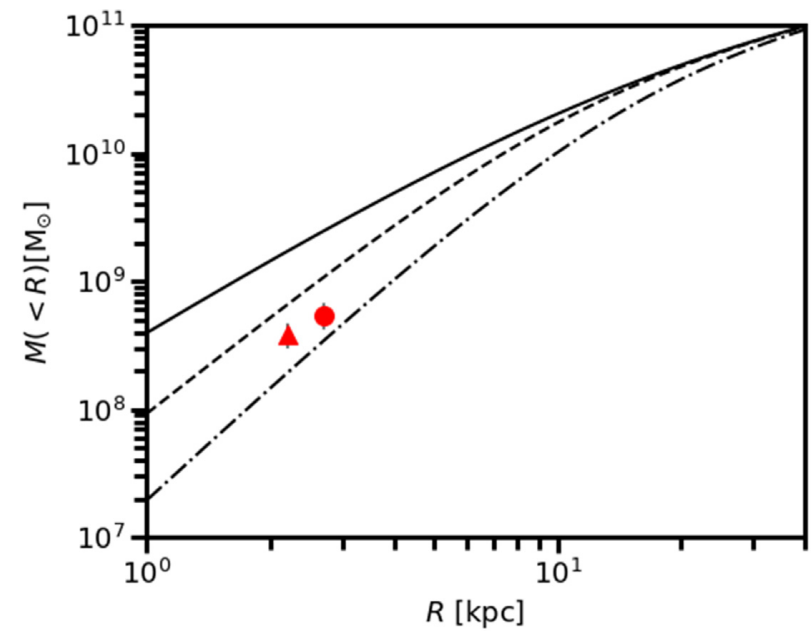

Figure 5. Halo mass profiles. Three halo mass profiles (Navarro et al. 1996, solid; Di Cintio et al. 2014, dashed; and Burkert 1995, dot-dashed) corresponding to a total halo mass of $\sim 2 \times 10^{11} \mathrm{M}_{\odot}$ are shown. The half mass (derived from integrated stars) and the TME mass (derived from globular cluster orbits) are shown as the red circle and the red triangle, respectively. The Di Cintio et al. (2014) and Burkert (1995) mass profiles with cores are more consistent than a cuspy NFW profile with our dynamical mass measurements.

In order to search for rotation in the stellar body of NGC 5846_UDG1, we have split our KCWI data set into two parts, with data coming from the NE and SW regions of the galaxy (see Fig. 1 illustrating the two regions). Each part has an $\mathrm{S} / \mathrm{N} \sim 16$ $\AA^{-1}$ and again we mask out the two bright GCs. We effectively partition the galaxy across a position angle of $150^{\circ}$. Our position angle lies within the range of the rotation axis of the GC system as determined by $\mathrm{M} 20$, i.e. $110 \pm 50^{\circ}$. We note that visual inspection of a simple 2D velocity map of the GCs relative to the host galaxy systemic velocity does not reveal an obvious rotation axis.
From the NE region of the galaxy, we measure a recession velocity of $2166 \pm 2 \mathrm{~km} \mathrm{~s}^{-1}$ and from the SW region $2171 \pm 2 \mathrm{~km} \mathrm{~s}^{-1}$. Thus, we find little, or no, evidence for rotation along this axis. We also measure similar velocity dispersions in the two regions, both of which are consistent with the overall value we measure (see Table 1). We note that the best-studied UDG, with a radial kinematic profile, is that of DF44 in the Coma cluster. In this case, rotation of the stellar body can be strongly ruled out (van Dokkum et al. 2019a). A general lack of rotation in the UDG population would argue against formation mechanisms that predict rotation in UDGs (Amorisco \& Loeb 2016; Cardona-Barrero et al. 2020; Wright et al. 2020).

\section{CONCLUSIONS}

In this work, using spectra from KCWI, we reconfirm membership of the NGC 5846 group and measure the stellar velocity dispersion for the UDG NGC 5846_UDG1. Our stellar velocity dispersion of $\sigma_{*}=$ $17 \pm 2 \mathrm{~km} \mathrm{~s}^{-1}$ is consistent within the uncertainty of the previously published value based on a dozen GCs (i.e. $\sigma_{\mathrm{GC}}=9.4_{-5.4}^{+7.0} \mathrm{~km} \mathrm{~s}^{-1}$ ). However our value, with a lower uncertainty, translates into a massto-light ratio that indicates a significant fraction of dark matter within the deprojected half-light radius. Applying the TME to the GC system also suggests the presence of dark matter at small radii.

We compare velocity dispersions derived from GC systems and integrated stars for pressure-supported galaxies from the literature, finding that the current data are generally consistent with a one-toone relation. However, given the relatively small number of GCs associated with a given UDG and various systematic uncertainties present (e.g. orbits, inclination, rotation), we favour the use of integrated stars to probe UDG kinematics and their dynamical masses.

By dividing our KCWI data into two regions along the NE-SW direction, we test for bulk rotation in the galaxy, finding none. The galaxy appears to be dominated by random motions. This argues against formation mechanisms that predict clear rotation in UDGs. 
We also measure the recession velocity for the two brightest compact objects in our field of view (one of which may be the galaxy stellar nucleus given its brightness and central location). We find them both to have a similar velocity to that of the host galaxy.

Our previous deep imaging, and spectroscopy in the literature, revealed a populous GC system associated with NGC 5846_UDG1. We estimate a total GC system count of $\sim 45$. The scaling relation between the number of GCs and halo mass implies a total halo mass of $\sim 2 \times 10^{11} \mathrm{M}_{\odot}$. This suggests NGC 5846_UDG1 hosts an overly massive dark matter halo for its stellar mass $\left(1.1 \times 10^{8} \mathrm{M}_{\odot}\right.$. Given this total halo mass, Di Cintio et al. (2014) and Burkert (1995) mass profiles with cores are more consistent than a cuspy NFW profile with our dynamical mass measurements. Further studies are required to determine the overall frequency of UDGs with overly massive haloes, whether they are non-rotating and if they lie within cored dark matter haloes.

\section{ACKNOWLEDGEMENTS}

We thank E. Iodice for helpful comments and S. Danieli for help with the observations. DAF thanks the ARC for financial support via DP160101608. AFM has received financial support through the Postdoctoral Junior Leader Fellowship Programme from La Caixa Banking Foundation (LCF/BQ/LI18/11630007). AJR was supported by National Science Foundation grant AST-1616710 and as a Research Corporation for Science Advancement Cottrell Scholar.

This work was partially supported by a NASA Keck PI Data Award, administered by the NASA Exoplanet Science Institute. The data presented herein were obtained at the W. M. Keck Observatory, which is operated as a scientific partnership among the California Institute of Technology, the University of California, and the National Aeronautics and Space Administration. The Observatory was made possible by the generous financial support of the W. M. Keck Foundation. The authors wish to recognize and acknowledge the very significant cultural role and reverence that the summit of Maunakea has always had within the indigenous Hawaiian community. We are most fortunate to have the opportunity to conduct observations from this mountain.

\section{DATA AVAILABILITY}

The data are available via the Keck Observatory Archive (KOA): https://www2.keck.hawaii.edu/koa/public/koa.php 18 months after observations are taken.

\section{REFERENCES}

Alabi A. B. et al., 2016, MNRAS, 460, 3838

Amorisco N. C., Loeb A., 2016, MNRAS, 459, L51
Beasley M., 2016, ApJL, 819, L20

Burkert A., 1995, ApJ, 447, L25

Burkert A., Forbes D. A., 2020, AJ, 159, 56

Cappellari M., 2017, MNRAS, 466, 798

Cardona-Barrero S., Di Cintio A., Brook C. B. A., Ruiz-Lara T., Beasley M. A., Falcón-Barroso J., Macciò A. V., 2020, MNRAS, 497, 4282

Carleton T., Errani R., Cooper M., Kaplinghat M., Peñarrubia J., Guo Y., 2019, MNRAS, 485, 382

Coelho P., 2014, Rev. Mex. Astron. Astrofis. Conf. Ser., 44, 151

Conroy C., Villaume A., van Dokkum P. G., Lind K., 2018, ApJ, 854, 139

Danieli S., van Dokkum P., Conroy C., Abraham R., Romanowsky A. J., 2019, ApJ, 874, L12

de Blok W. J. G., Bosma A., 2002, A\&A, 385, 816

Di Cintio A., Brook C. B., Dutton A. A., Macciò A. V., Stinson G. S., Knebe A., 2014, MNRAS, 441, 2986

Fahrion K. et al., 2020, A\&A, 637, A26

Forbes D. A. et al., 2011, MNRAS, 413, 2665

Forbes D. A. et al., 2019, A\&A, 626, A66

Forbes D. A. et al., 2020, MNRAS, 492, 4874

Gannon J. S. et al., 2020, MNRAS, 495, 2582

Janssens S. R. et al., 2019, ApJ, 887, 92

Laporte C. F. P., Agnello A., Navarro J. F., 2019, MNRAS, 484, 245

Mahdavi A., Trentham N., Tully R. B., 2005, AJ, 130, 1502

Martín-Navarro I. et al., 2019, MNRAS, 484, 3425

Martin C. et al., 2010, in McLean I. S., Ramsay S. K., Takami H., eds, Proc. SPIE Conf. Ser., Vol. 7735, Ground-based and Airborne Instrumentation for Astronomy III . SPIE, Bellingham, p. 77350M

McConnachie A. W., 2012, AJ, 144, 4

Miller B. W., Lotz J. M., 2007, ApJ, 670, 1074

Müller O. et al., 2020, A\&A, 640, A106(M20)

Navarro J. F., Frenk C. S., White S. D. M., 1996, ApJ, 462, 563

Pota V. et al., 2013, MNRAS, 428, 389

Seigar M. S., 2011, ISRN Astron. Astrophys., 2011, 725697

Spavone M. et al., 2017, A\&A, 603, A38

Tully R. B. et al., 2013, AJ, 146, 86

van Dokkum P. G., Abraham R., Merritt A., Zhang J., Geha M., Conroy C., 2015, ApJ, 798, L45

van Dokkum P. et al., 2016, ApJ, 828, L6

van Dokkum P. et al., 2017, ApJ, 844, L11

van Dokkum P. et al., 2018, Res. Notes Am. Astron. Soc., 2, 54

van Dokkum P., Danieli S., Abraham R., Abraham R., Conroy C., Romanowsky A. J., 2019a, ApJ, 874, L5

van Dokkum P. et al., 2019b, ApJ, 880, 91

Wasserman A. et al., 2019, ApJ, 885, 155

Watkins L. L., Evans N. W., An J. H., 2010, MNRAS, 406, 264

Wolf J. et al., 2010, MNRAS, 406, 1220

Wright A. C. et al., 2020, preprint (arXiv:2005.07634)

This paper has been typeset from a $\mathrm{T}_{\mathrm{E}} \mathrm{X} / \mathrm{L} \mathrm{T}_{\mathrm{E}} \mathrm{X}$ file prepared by the author. 